\title{
EMI Signal Encoding based on Deep Auto-encoder Combined with Wavelet Transformation
}

\author{
Zhen Peng ${ }^{1}$, Hongyi $\mathrm{Li}^{1}$, Shengyu Chen ${ }^{1}$ and Di Zhao ${ }^{1,+}$ \\ ${ }^{1}$ LMIB, School of Mathematical Sciences, Beihang University, Beijing 100191, China
}

\begin{abstract}
With the development of electronic technology, digital devices are becoming more sensitive to electromagnetic interference. As a result, the recognition methods are under scrutiny. To find the way to improve the accuracy of electromagnetic interference recognition, this paper presents a signal encoding method based on deep auto-encoder and wavelet transformation. We use wavelet de-noising as the first step on our method to reduce the noise interference. Then a deep auto-encoder is trained for extracting EMI signals' feature. Results on evaluation the reconstruction error and recognition accuracy demonstrate that our approach outperforms comparison methods, which indicates that the proposed method could better capture the data structure of the high dimensional EMI signals.
\end{abstract}

Keywords: EMI signal, wavelet transform, signal dimension reduction, auto-encoder.

\section{Introduction}

Due to the wide application of high precision electronic equipment, the issue of electromagnetic compatibility (EMC) has been increasingly attracting public attention in recent years. In practice, electromagnetic interference (EMI) signals are usually high-dimensional and non-stationary, which makes the analysis and identification of EMI signals a very challenging problem. Therefore, effective dimension reduction techniques are desired in order to gain a promising recognition performance.

Many works have been proposed to addressed the signal dimensionality reduction problem [1-4], such as principal component analysis (PCA), multidimensional scaling (MDS), isometric mapping (ISOMAP) and locally linear embedding (LLE). Besides, noisy signal components would deteriorate the encoding accuracy and classification result. To address those limitations, we propose a novel signal encoding method suitable for high dimension EMI signals. Combined with wavelet transformation de-noising, our method encodes EMI signal by a deep auto-encoder, which extracts representative low dimensional features from signals.

The remainder of this paper is organized as follows: in the next section, related works in recently years are discussed. In section 3, the proposed method will be depicted in detail. And in section 4, comparison experiments are demonstrated, followed by a conclusion in the last section.

\section{Related Work}

The essence of wavelet transform is to reduce or completely eliminate the coefficients produced by noise, at the same time, to maximize the retention of coefficients of real signal. The basic steps are shown in Fig. 1.

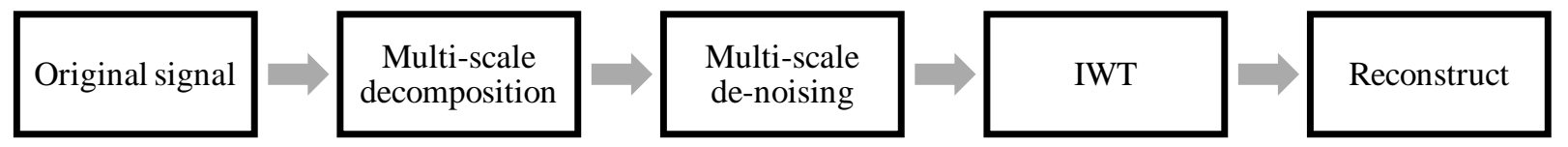

Fig. 1: The process of wavelet transforms de-noising

Deep auto-encoder consists of encoder, decoder and hidden layer. The values of hidden layer neurons, called the encoding, are obtained via eq. (1), where $x$ is input vector, $S$ denotes sigmoid function, $b$ is the vector of hidden neuron biases, and matrix $W$ is hidden weights. The values of output neurons, called the decoding, are computed as in eq. (2), where vector $c$ is output neuron biases. Unsupervised learning of

\footnotetext{
+ Corresponding author.

E-mail address: zdhyl2010@163.com.
} 
weights and biases of auto-encoders can be achieved by gradient descent, based on a training set of input vectors.

The values of output neurons, called the decoding, are computed as in eq. (2), where vector $c$ is output neuron biases. Unsupervised learning of weights and biases of auto-encoders can be achieved by gradient descent, based on a training set of input vectors.

$$
\begin{gathered}
h(x)=S(W x+b) \\
\hat{x}(h(x))=S\left(c+W^{T} h(x)\right)
\end{gathered}
$$

The training process of the deep auto-encoder contains pre-training and fine-tuning. The training process is mainly based on [5], which uses a training method based on RBM [6-7]. See more details in section 3.2.

This paper combines wavelet transformation with deep auto-encoder, applying to the EMI signal encoding. As a result, wavelet transformation will reduce the effect from noise signal on feature extracting so that we can get codes with less noise information through deep auto-encoder.

\section{Signal EncodingCombined with Wavelet Transform}

In this section, we will describe the details of the proposed method.

\subsection{Signal De-noising}

Considering the influence of noises on the performance of EMI signal encoding, this paper presents a new EMI signal encoding method based on wavelet transformation and deep auto-encoder. The first step of our approach is to reduce the signal noises with two assumptions.

1) Smoothness: generally, the de-noised and original signal should at least have the same smoothness.

2) Similarity: the variance of the de-noised signal and the original signal is estimated to be the minimum variance in the worst case (Minimax Estimator) [8].

A one-dimensional signal containing noise model can be expressed as the following form:

$$
S(t)=f(t)+\sigma \cdot e(t), t=0,1, \cdots, n-1
$$

where $f(t)$ is the real signal; $e(t)$ represents the noise; $S(t)$ is the signal with noise. In practice, the most desired signals are usually stable signals, or signals with low frequencies, considering that noisy signals are usually of high frequencies. This difference between noise signals and desired signals provides the possibility using wavelet transformation. While doing wavelet decomposition, noise is mainly contained in the high frequency wavelet coefficients. As a result, we can set the threshold to wavelet coefficients and reconstruct it to achieve the purpose of noise eliminating. The aim of de-nosing is to suppress the noise signals of $S(t)$, resulting in recovering the true signal $f(t)$.

De-noising steps using wavelet are as follow:

(1) Pre-processing to facilitate subsequent processing.

(2) One-dimension signal wavelet decomposition. Choose the number of wavelet decomposition layer $N$, and decompose the signal to $N$ layer.

(3) Threshold quantization of high-frequency coefficients of wavelet decomposition. To those highfrequency coefficients in every layer, choose a threshold and do soft or hard threshold quantization.

(4) Reconstruction of one-dimensional signal through wavelet. According to the coefficients of the $N$ th layer and quantized high-frequency coefficients from layer 1 to layer $N$, reconstruct the signal.

From the above 4 steps can be seen, the key is how to select the threshold and how to carry out the threshold quantization. To some extent, it is directly related to the result of signal de-noising.

\subsection{Deep Auto-encoder}

After signal de-noising, the reconstructed signal retains the main characteristic of the EMI signal. As reconstructed signals the input vector, we then train the RBM nets. Taking the RBM weight vector as an initial value assigned to the deep auto-encoder after training.

(1) Abbreviations and Acronyms 
We first train a Gaussian-binary Restricted Boltzmann Machine (GB-RBM) [5, 9]. It contains a visible layer and a hidden layer. The hidden layer has 500-3000 latent variables. There is full connectivity between layers, but no connections within either layer. The connection weights (and biases) can be learned efficiently using the contrastive divergence approximation to the log likelihood gradient. For a GB-RBM, the conditional density of a visible vector, $o$, given a hidden binary vector, $h$, is

$$
p(o \mid h)=N\left(o ; b+h^{T} W^{T}, I\right)
$$

and the element-wise conditional distribution of $h$ given $o$ is

$$
P\left(h_{j}=1 \mid o\right)=\sigma\left(c+o^{T} W\right)
$$

where $\sigma(x)=\left(1+e^{-x}\right)^{-1}$. Two conditional distributions can be shown to correspond to the generative model.

$$
p(o, h)=\frac{1}{z} \exp (-E(o, h))
$$

where $E(o, h)=(o-b)^{T}(o-b) / 2-c^{T} h-o^{T} W h$, and the partition function $Z$ is the normalization factor.

After introducing the GB-RBM we treat the activation probabilities of its hidden units, when they are being driven by data, as the data for training a binary-binary RBM (BB-RBM) [6]. These two RBM's can then be composed to form a deep belief net (DBN) in which it is easy to infer the states of the second layer of binary hidden units from the input in a single forward pass $[6,10]$. The DBN used in this work is illustrated on the left side of Fig. 2, where the two RBMs are shown in separate boxes.

The binary states, which are the hidden units of the top RBM, form the code for the input. Even lower distortion can be achieved by a further step of fine-tuning which we describe now.

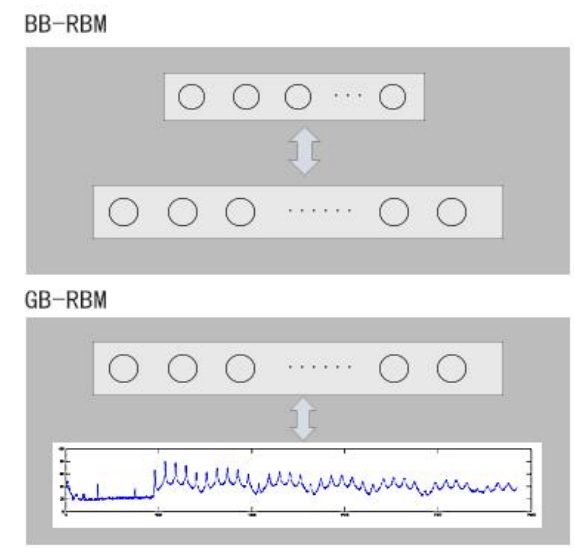

Fig. 2: Illustration of pre-training of the DBN that consists of two RBMs used in this work.

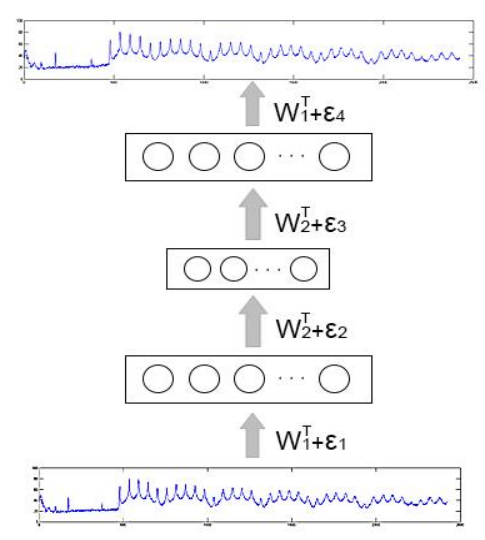

Fig. 3: Illustration of fine tuning that produces the final deep auto-encoder.

\section{(2) The Construction of the Auto-encoder}

To fine-tune, we first "unroll" the DBN by using its weight matrices to create a deep, five-layer network whose lower layers use the matrices to encode the input and whose upper layers use the matrices in reverse order to decode the input. This auto-encoder is then fine-tuned using back-propagation of error-derivatives to make output as similar as possible to input, as shown in Fig. 3. The conjugate gradient is used for the finetuning. Details of the training process, including the division of the training set into mini-batches, number of training passes (epochs) in pre-training and fine-tuning, learning rate, momentum, weight decay, and the threshold used to force binary codes, etc., are found to be important to obtain good encoding results [11-13].

After both pre-training and fine-tuning, we encode and reconstruct any variable-length spectrogram as follows. Firstly, $N$ consecutive overlapping frames of 256-point log power spectra are each normalized to zero-mean and unit-variance to provide the input to the auto-encoder. The first hidden layer then uses the logistic function to compute real-valued activations. These real values are fed to the next, encoding layer to compute "codes". The real-valued activations of hidden units in the encoding layer are quantized to be either zero or one with 0.5 as the threshold. These binary codes are then used to reconstruct the original 
spectrogram, where individual fixed-frame patches are reconstructed first using the two upper layers of network weights. Finally, the overlap-and-add technique is used to reconstruct the full-length speech spectrogram from the outputs produced by applying the auto-encoder to every possible window of $N$ consecutive frames.

\section{Experiments Results and Analysis}

To compare PCA and deep auto-encoder in EMI signal dimension reduction and noise reduction, we first test PCA, deep auto-encoder and deep auto-encoder combined with wavelet transform on the same signal datasets. We select 150 groups of real EMI signals, and every group of signal contains 2423-dimensional frequency domain data. All the signals are set as training data, and we set the compression dimension number (in experiments we compress signals into 50 or 100 dimension). Then we reconstruct the signal and calculate the bias between reconstructed signal and original signal, which reflects the compression reconstruction effect.

When using deep auto-encoder, we need to know the number of hidden units after we set the number of encoding units. According to [5], we set it to be 500, 750 and 1000 in our experiments. We use db3 wavelet in signal de-noising and decompose the signal into the third layer, which ensures that the signal reduces enough noise but do not lose the characteristics the real signal contains. After encoding and reconstruction to those 150 groups of EMI signals, the average reconstruction error is calculated as shown in Table 1. "Auto" represents the results using deep auto-encoder and "w-Auto" means using wavelet transform de-noising before encoding. Each method encodes signal to a specific dimension "d" shown in following brackets.

Table 1: Comparison of PCA, LLE, MDS, ISOMAP and deep auto-encoder on the EMI signal encoding errors

\begin{tabular}{|c|c|c|c|c|c|c|c|c|c|c|}
\hline \multirow{2}{*}{ Encoding method } & \multirow{2}{*}{ PCA } & \multirow{2}{*}{ LLE } & \multirow{2}{*}{ MDS } & \multirow{2}{*}{ ISOMAP } & \multicolumn{4}{|c|}{ Auto } & \multicolumn{3}{|c|}{ W-Auto } \\
\cline { 6 - 11 } & & & & & 500 & 750 & 1000 & 500 & 750 & 1000 \\
\hline$d=50$ & 1.1205 & 0.6118 & 0.6524 & 0.6872 & 0.6921 & 0.6351 & 0.6402 & 0.6502 & 0.5949 & 0.6008 \\
\hline$d=100$ & 0.4687 & 0.0682 & 0.0892 & 0.1039 & 0.0803 & 0.0575 & 0.0619 & 0.0753 & 0.0531 & 0.0566 \\
\hline
\end{tabular}

It is obviously seen that de-noising using wavelet transformation before feature extraction will notably reduce the reconstruction errors. The reduction of noise component in the encoding result will make the reconstructed signal closer to the original one. Results also shows that it's important to choose the number of hidden units, which may rely on the number of input and output units.

The reconstruction error on EMI signal compression to the dimension 100 by PCA and deep autoencoder are shown in Fig. 4 From top to bottom are reconstruction errors of PCA, deep auto-encoder with 500 hidden units and deep auto-encoder with wavelet de-noising.
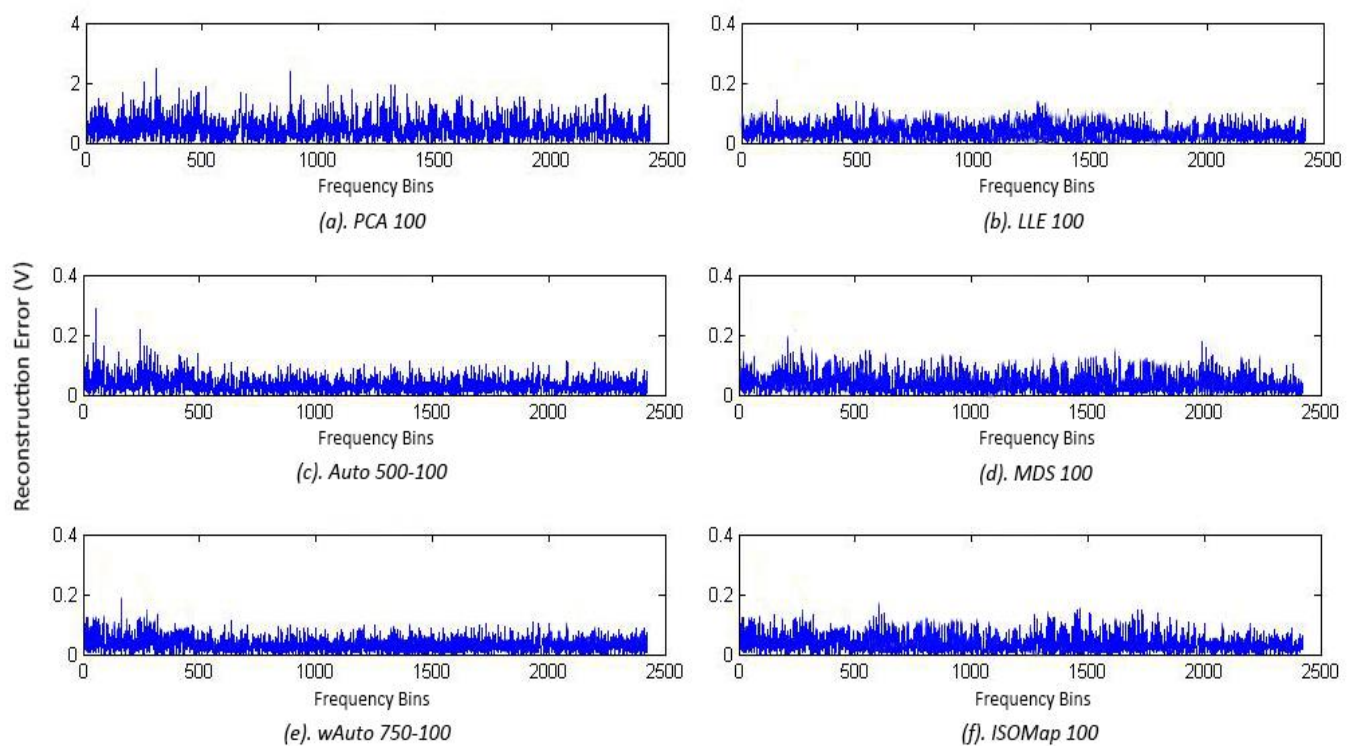

Fig. 4: The errors of reconstruction on the EMI signal compression to 100-dimension by PCA, deep auto-encoder, LLE, MDS and ISOMAP 
To further validate the encoding efficiency, classification experiments are carried out to compare a variety of dimension reduction or encoding methods on EMI signal. We use k-NN algorithms as the baseline classifier. All the 150 groups of EMI signals (with class labels) are encoded into 50-dimension in different methods separately in 10-fold cross-validation. In other words, data sets are divided into ten parts, 9 of which are used for training and the rest are used for testing. We report the mean recognition accuracy after 10 runs. We choose the best result of each method in different training frequency as the final accuracy result as shown in Table 2:

Table 2: Accuracy of different methods on the EMI signal encoding classification

\begin{tabular}{|c|c|c|c|c|c|c|}
\hline Compression method & w-Auto-encoder & Auto-encoder & PCA & LLE & MDS & ISOMAP \\
\hline Accuracy & $66.32 \%$ & $63.57 \%$ & $51.61 \%$ & $60.29 \%$ & $58.92 \%$ & $57.84 \%$ \\
\hline
\end{tabular}

Compare to traditional dimension reduction methods, such as PCA, the proposed deep auto-encoder has a better EMI signal encoding capability, which means our method has a better ability to learn and extract EMI features. In addition, to the problem that how to decide the number of hidden units, we try using 500, 750 and 1000 hidden units on data compression. A better number of hidden units depends on the dimension of data, data structure, and the compression dimension. In particular, we find that setting 750 hidden units are the best choice in classification. More or less hidden units may result in network insufficient learning or over-fitting, which increases reconstructing errors. Furthermore, de-noising by wavelet transform help deep auto-encoder extract signal feature and increase the accuracy of classification which depends on signal feature extraction.

\section{Conclusions}

This paper presents an EMI signal encoding method based on deep auto-encoder and wavelet transform. We compare this method with traditional dimension reduction methods such as PCA, LLE, MDS and ISOMAP on the EMI signal dimension reduction. The experiment results on evaluation of the reconstruction error and recognition accuracy demonstrate that our approach outperforms comparison methods, which indicates that the proposed method could better capture the data structure of the high dimensional EMI signals. Future work includes improving the robustness of deep auto-encoder to noise and outliers.

\section{Acknowledgements}

This work was supported by the National Natural Science Foundation of China (Grant no. 61771001).

\section{References}

[1] D. Zhao, H. Lu, H. Li. An ICA and AIS Based Method for Electromagnetic Compatibility Analysis. Advanced Materials Research, vol. 446-447, pp. 547-550, 2013.

[2] H. Li, M. Ye, D. Zhao. An improved ICA algorithm based on the negative entropy and simulated annealing algorithm. Applied Mechanics and Materials, vol. 411-414, pp. 1125-1128, 2013.

[3] D. Zhao, H.F. Liu, H.Y. Li. A Method Based on Grey Correlation Clustering for Improving the Hierarchy of Analytic Hierarchy Process. in Proc. IEEE/WIC/ACM International Conference on WI-IAT, vol. 3, pp. 156-159, 2010.

[4] H.Y. Li, Y. Fu, D. Zhao. Identification of Power Quality Disturbances Based on FFT and Attribute Weighted Artificial Immune Evolutionary Classifier. Applied Mechanics and Materials, vol.530-531, pp.277-280, 2014.

[5] L. Deng, M.L. Seltzer, D. Yu, et al. Binary coding of speech spectrograms using a deep auto-encoder. Interspeech, pp. 1692-1695, 2010.

[6] G. Hinton, S. Osindero, Y. The. A fast learning algorithm for deep belief nets. Neural Computation, vol. 18, pp. 1527-1554, 2006.

[7] G. Hinton, R. Salakhutdinov. Reducing the dimensionality of data with neural networks. Science, vol. 313. no. 5786, pp. 504 - 507, 2006.

[8] F. Yang. Wavelet analysis and application in Engineering. Science Press, pp. 132-139, 1999.

[9] Melchior J, Wang N, Wiskott L. Gaussian-binary restricted Boltzmann machines for modeling natural image 
statistics. PLOS ONE, vol. 12, pp. e0174289, 2017.

[10] X. He, L. Deng, W. Chou. Discriminative Learning in Sequential Pattern Recognition-A Unifying Review for Optimization-Oriented Speech Recognition. IEEE Sig. Proc. Mag., vol. 25, pp. 14-36, 2008.

[11] D. Mishra, S.K.Singh, R.K.Singh. Wavelet-Based Deep Auto Encoder-Decoder (WDAED)-Based Image Compression. IEEE Trans. Circuits Syst. Video Technol. vol. 31(4), pp. 1452-1462, 2021.

[12] Q. Zhu, H. Wang, R. Zhang. Wavelet Loss Function for Auto-Encoder. IEEE Access. vol. 9, pp. 27101-27108, 2021.

[13] H. Shao, H. Jiang, X. Li, et al. Intelligent fault diagnosis of rolling bearing using deep wavelet auto-encoder with extreme learning machine. Knowl. Based Syst. vol. 140, pp. 1-14, 2018. 